\title{
Successful Salvage of a Mangled Injury of the Ankle in a Young Male
}

\author{
Abhishek Vaish ${ }^{1}$, Ashish Goyal ${ }^{1}$, Raju Vaishya ${ }^{1}$
}

Learning Point of the Article:

A mangled injury of the distal leg and ankle can be salvaged in young individuals, if timely and appropriate surgical treatment can be done by a team of Orthopedic and Plastic surgeons.

\section{Abstract}

Introduction: The salvage of a mangled limb is quite challenging, but can be rewarding especially for younger individuals.

Case Report: We present a case of mangled injury of the ankles in a young male, which was salvaged successfully, using Orthopaedic and Plastic Surgery procedures.

Conclusion: The GHSS is helpful for decision-making and to predict the ultimate outcome of the open injuries. Younger patients with intact vascularity of the limb should be considered for limb salvage with a multidisciplinary stepwise approach.

Keywords: Mangled extremtity, Lower limb, Ankle, Plastic Surgery, Arthrodeis, Fractures

\section{Introduction}

According to a dictionary definition, the verb "mangle" means to "destroy or severely damage by tearing or crushing." A mangled extremity is characterized by injury to multiple musculoskeletal parts such as the bone, muscle, vascular system, nerves, and soft tissue, around the extremity. It usually occurs in severe traffic accidents (road traffic accident [RTA]) and bomb blast injuries and the patients usually present with severe compound fractures with significant soft-tissue loss. A difficult choice between amputating and salvaging the limb is to be made in these cases $[1,2]$. The available scoring systems for open fractures are not entirely reliable to treat mangled extremities. Besides, it remains controversial whether amputation or limb salvage is the best approach because there are no clear criteria available for treatment. If limb salvage is selected, the goal is to reconstruct the limb to enable the patient to walk without pain and deformity. To achieve this, the surgical team must include experienced orthopedic and plastic surgeons and a rehabilitation specialist. Choosing an appropriate reconstruction method and its timing is crucial to a successful outcome. We describe a young male with a mangled leg who was successfully treated with limb salvage and went on to achieve a good outcome.

\section{Case Report}

A 19-year-old young man, with no underlying disease, sustained a crush injury to his leg and ankle in a motorbike crash in RTA. He had sustained open fractures of the right leg and ankle bones (Gustilo-Anderson classification [GAC] Type III-B)[3]. His right distal tibia, fibula, and talus bones were crushed, and the large parts of the bones were protruding out from the wound (Fig. 1).

There was no damage to important internal organs, such as the brain or heart, but he was hypotensive and responded to fluids and blood transfusions. The Mangled Extremity Severity Score (MESS) was $7[3,4]$, and the Ganga Hospital Scoring System (GHSS) [5] was 12 on arrival. The patient was advised for a below-knee amputation based on clinical judgment, poor financial status, risk of sepsis, and expected multiple surgeries.

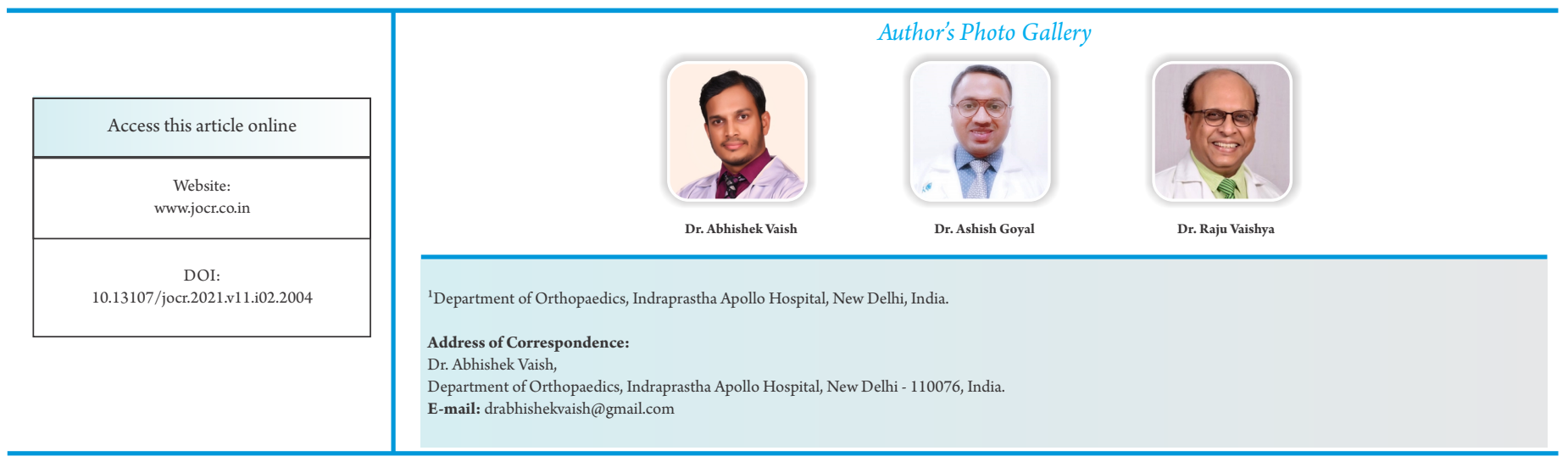

Journal of Orthopaedic Case Reports | pISSN 2250-0685 | eISSN 2321-3817 | Available on www.jocr.co.in | doi:10.13107/jocr.2021.v11.i02.2004 This is an Open Access article distributed under the terms of the Creative Commons Attribution Non-Commercial License (http://creativecommons.org/licenses/by-nc/3.0) which permits unrestricted non-commercial use, distribution, and reproduction in any medium, provided the original work is properly cited. 


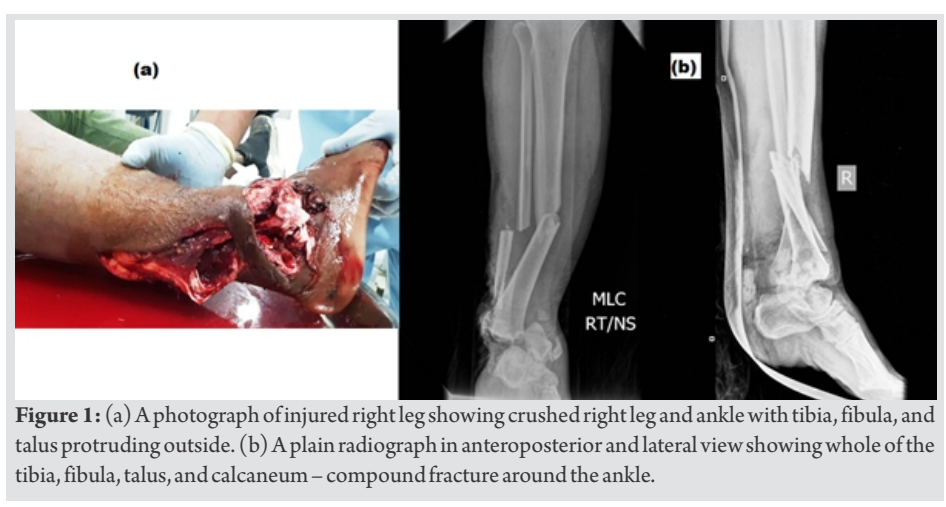

However, the family and the patient did not agree for it and wanted us to proceed with a trial of salvaging the limb, considering his young age.

\section{Investigations}

Plain radiographs of the right leg with ankle joint (anteroposterior and lateral views) were taken. It revealed dislocation of the ankle joint, comminuted fracture of the distal fibula, distal tibia, and talus with gross ankle instability (Fig. 1). His hemoglobin was $7.0 \mathrm{~g} / \mathrm{dl}$, on admission, and the other blood parameters were normal.

\section{Treatment}

The patient was first stabilized and then taken to the operation theater where extensive wound debridement with removal of necrotic tissue and bone was performed. The leg and ankle were stabilized with abiplanar external fixator, and the highly unstable ankle joint was stabilized with a $3 \mathrm{mmKirschner}(\mathrm{K})$ wire. The soft tissue was handled very carefully and wound defect was loosely approximated (Fig. 2).

The open wounds were subsequently managed with negative pressure wound therapy. After several repeated debridement, when the condition of the wound was improved, split-thickness skin grafting was done and soft-tissued efect was finally covered and healed in 2 months' time. After complete healing of the wounds, the external fixator was removed at 3 months, and an above-knee cast was applied (Fig. 3 ).

The displacement of tibial shaft fracture and the ankle subluxation persisted in the cast, and the fracture did not progress to union. Hence, after 20 weeks from the time of injury,

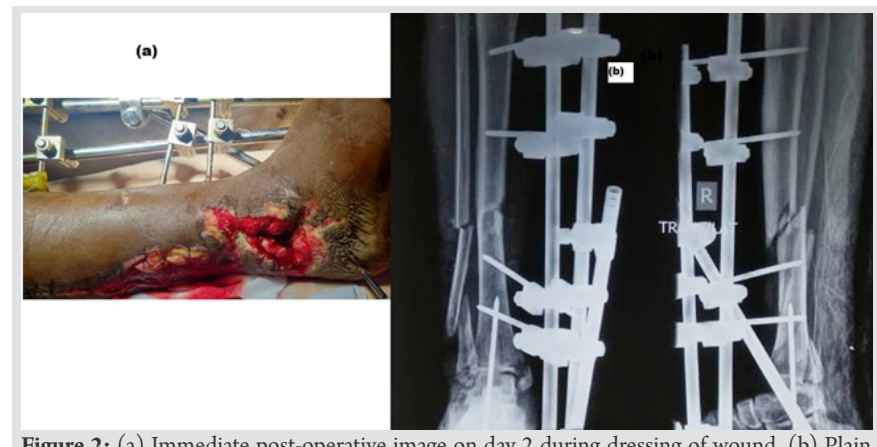

Figure 2: (a) Immediate post-operative image on day 2 during dressing of wound. (b) Plain radiograph showing fracture fixation with external fixators and K-wire.

a hindfoot arthrodesis with fixation of tibial fracture was done by a $250 \times 10 \mathrm{~mm}$ long retro grade inter locking nail(Smith and Nephew)of the tibia through the calcaneum. The tibial shaft fracture could not be adequately fixed with this nail and hence an additional augmentation plate was applied to fix the tibial fracture (Fig. 4).

\section{Outcome and follow-up}

The wounds and the fracture healed without any significant problems at 6 months after the injury. The patient had a plantigrade foot, with good soft-tissue coverage around the ankle and the leg (Fig. 5). The patient could walk well without any pain and support at 1 year follow-up.

\section{Discussion}

Several scoring systems have been suggested for evaluating the severe compound injuries of the extremities. These aim to guide the surgeons regarding appropriate management and likely outcomes of the treatment. However, no exclusive scoring system has yet been established regarding the management of a mangled extremity. The GAS Type-IIIB classification of open fractures, like in this case, includes a wide spectrum of injuries (from the easily manageable to the barely salvageable) and is, therefore, unable to provide accurate guidelines for the management or allow comparison of published results from differentinstitutions.

The MESS recommends a primary amputation in injuries when the MESS score is $\geq 7[3,4]$. Absolute indications for primary amputation of the mangled lower extremity are complete anatomical disruption of the posterior tibial nerve or crush

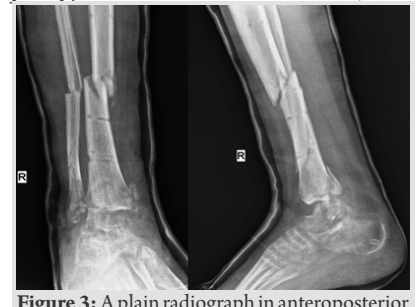

Figure 3: A plain radiograph in anteroposterior and lateral view, 2 months post-operative showing distal end of tibia, fibula, and ankle joint with cast insitu.

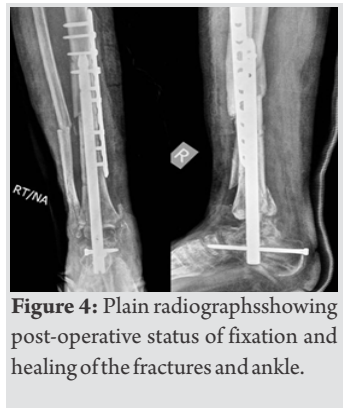

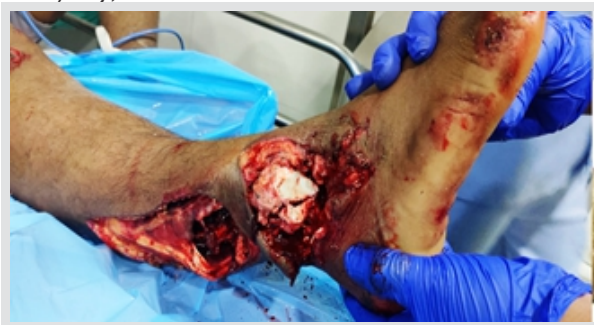

Figure 5: Immediate status of wound at time of injury and final outcome after 6 months of the injury. 
injuries with a warm ischemia time longer than $6 \mathrm{~h}$. These indications did not apply to the current patient. Due to the patient's young age, a borderline GHSS of 12 , and persistent requests we opted to perform the limb salvage, as the preferred treatment.

In this case, the most important factors for successful salvage were the timing and method of reconstruction of soft tissues and bones. Ellington et al. [6] reported no significant differences between the amputation group and the salvage group in terms of pain, walking speed, and return-to-work rate at 2 years, despite a longer time to full weight-bearing in the salvage group. Despite a borderline MESS and GHSS, due to the overall health status and the young age of the patient and local clinical status, reconstruction was attempted. After 3 months of treatment, all wounds have healed completely, and satisfactory motor and sensory function were achieved. At 6 months, the fractures were united with good hindfoot arthrodesis, with a painless, plantigrade foot. This case was a true representation of a traumatic mangled extremity, which literally means "to damage something so badly that it is difficult to see what it looked like originally," but the salvage and outcomes were highly satisfactory and beyond expectations.

Several classifications for open fractures and injury severity scores are known $[1,2,5]$ and have been developed to guide the surgeon in making appropriate decisions. The first decision in the management of a severely injured lower limb is the question of salvage. While it would be a disaster to amputate a salvageable limb, failed reconstructions leading to secondary amputations are unacceptable and frustrating to the patient. There are numerous scoring systems developed for the management of the mangled extremity aside from the MESS. Other scoring systems include the predictive salvage index, limb salvage index, nerve injury, ischemia, soft-tissue injury, skeletal injury, shock and age of patient score, and GAS. Studies have compared scoring systems, including MESS, and have failed to prove their validity $[4,7,8]$.

The most popular and commonly used GAS classifies type III open injuries into three grades; type III-A, where there is an adequate soft-tissue cover over the fracture, in spite of extensive skin loss, type III-B, where in addition to the extensive softtissue loss, there is periosteal stripping and exposure of the bone present, and the type-IIIC injuries include an open fracture with an associated arterial injury requiring repair $[3,4]$.The GHSS is more elaborate for type III-A and III-B type of injuries and allocates 1-5 points, according to the severity of injury to the (a) covering tissues (skin and fascia), (b) skeleton (bones and joints), and (c) functional tissues (muscles, tendons, and nerves). Furthermore, it takes care of the systemic factors which may influence treatment and the outcome is given 2 points each and the final score is arrived at by adding the individual scores together [5]. A score of 14 was found to be the threshold for an amputation. Based on GHSS, it is recommended that the injuries with a score of 14 and below should have attempted at salvage; those with a score of 17 should go for primary amputation of the limb.

The GAS has limitations as regards the management of Type III-B compound injuries, as its focus is only on the soft- tissue injury, whereas in such injuries, the skin, muscles, nerves, and bones are injured to varying degrees. Gustilo et al. reported that for the Type III open fractures (severe soft-tissue injury, segmental fracture, or traumatic amputation), the infection rates were $44 \%$ in the retrospective study and $9 \%$ in the prospective study, Brumback et al. [9] reported that interobserver agreement in the classification of open fractures of the tibia for each fracture ranged from 42 to $94 \%$ and the average agreement in the subgroup of surgeons who were considered to have the least experience (residents and fellows) was 59\% (range, 33-94\%) [10]. Hence, the degree of damage to the bone and muscles is not fully represented in this classification (GAC) and it may influence the final outcome. The GHSS was, therefore, introduced from Coimbatore, India, by Rajasekharan et al., which is a simple method of application and is reliable in predicting the prognosis for the limb salvage and outcome measures in type-IIIA and type-IIIB open injuries of the tibia. This score compares well with the MESS, both being highly sensitive (98\% and $99 \%)$, specific (100\% and $17 \%)$, with a positive predictive value of $100 \%$ and $97.5 \%$, and the negative predictive value of $(70 \%$ and $50 \%)$, respectively[5].

We found GHSS to be the most reliable than MESS and GAS scoring system to predict the need for primary amputation and the final outcome of the mangled extremity. In our case, the GHSS was 12, which suggests an attempt at salvage and the score 7 of MESS, points toward a primary amputation.

\section{Conclusion}

The GHSS is helpful for decision-making and to predict the ultimate outcome of the open injuries. It can also more accurately predict the need for amputation, even when the vascularity is not compromised, than the other score scoring systems $[3,4,5]$.

Younger patients with intact vascularity of the limb should be considered for limb salvage with a multidisciplinary stepwise approach. 
Clinical Message

The salvage of a mangled injury of the limb is quite challenging. Several scoring systems are described to classify these open injuries and to guide surgical management. We found GHSS, to be more reliable. The younger patients with intact vascularity of the limb should be considered for limb salvage (with GHSS <14). It is essential to involve an experienced plastic surgery team, from the beginning of the treatment of these mangled injuries.

\section{References}

1. Bakota B, Kopljar M, Jurjević Z, Staresinić M, Cvjetko I, Dobrić I, et al. Mangled extremity-case report, literature review and borderline cases guidelines proposal. Coll Antropol2012;36:1419-26.

2. Patel MB, Richter KM, Shafi S. Mangled extremity: Amputation versus salvage. Curr Trauma Rep 2015;1:45-9.

3. McNamara MG, Heckman JD, Corley FG. Severe open fractures of the lower extremity: A retrospective evaluation of the mangled extremity severity score (MESS). J Orthop Trauma 1994;8:81-7.

4. Scalea TM, DuBose J, Moore EE, West M, Moore FA, McIntyre $\mathrm{R}$, et al. Western trauma association critical decisions in trauma: Management of the mangled extremity.J Trauma Acute Care Surg 2012;72:86-93.

5. Rajasekaran S, Nareshbabu J, Dheenadhayalan AP, Shetty SR, Sundararajan MK, Rajasabapathy S. A score for predicting salvage and outcome in Gustilo TYPE-IIIA and TYPEIIIB open tibial fractures. J Bone Joint Surg Br 2006;88:1351-60.
6. Ellington K, Bosse MJ, Castillo RC, MacKenzie EJ, LEAP Study Group. The mangled foot and ankle: Results from a 2-year prospective study.J Orthop Trauma 2013;27:43-8.

7. Schirò G, Sessa S, Piccioli A, Maccauro G. Primary amputation vs limb salvage in mangled extremity: A systematic review of the current scoring system. BMC Musculoskelet Disord 2015;16:372.

8. Fodor L, Sobec R, Sita-Alb L, Fodor M, Ciuce C. Mangled lower extremity: Can we trust the amputation scores? Int J Burns Trauma 2012;2:51-8.

9. Gustilo RB, Anderson JT. Prevention of infection in the treatment of one thousand and twenty-five open fractures of long bones: Retrospective and prospective analyses. J Bone Joint Surg Am 1976;58:453-8.

10. Gustilo RB. Interobserver agreement in the classification of open fractures of the tibia. The results of a survey of two hundred and forty-five orthopaedic surgeons. J Bone Joint Surg Am 1995;77:1291-2.
Conflict of Interest: Nil

Source of Support: Nil

Consent: The authors confirm that informed consent was obtained from the patient for publication of this case report

\section{How to Cite this Article}

Vaish A, Goyal A, Vaishya R. Successful Salvage of a Mangled Injury of the Ankle in a Young Male. Journal of Orthopaedic Case Reports 2021 February;11(2): 11-14. 